\title{
POST PARTUM HAEMORHAGE AMONG WOMEN DELIVERED AT MBEYA REFERRAL HOSPITAL IN 2008.
}

\section{BY John Stanford ( MD5 2009), School Of Medicine}

\section{ABSTRACT \\ OBJECTIVES}

To assess the prevalence, associated risk factors and outcome of post partum hemorrhage (PPH) among women delivering at the hospital setting.

\section{DESIGN}

Descriptive retrospective hospital based cross-section study

\section{RESULTS}

The targeted respondents were 344 pregnant women. Prevalence of PPH was $11.9 \%$ with re-admission rate of $41.5 \%$ and $12.2 \%$ deaths secondary to PPH where most of the women $(35.2 \%)$ were given blood transfusion after re-admission and surgery as an immediate measure taken. Also it has been found that increase in blood loss is statistically associated with delivery by LSCS. $(\mathrm{P}=0.02)$

Risk factors for PPH found in this study are low hemoglobin (HB), pregnancy induced hypertension $(\mathrm{PIH})$, trauma after delivery, history of birth before arrival (BBA), advanced age group ( $>32$ years old) and multiparity ( $>3$ parity).

\section{CONCLUSION}

Although the prevalence seems to be low as compared to overall for Africa (33.4\%), still PPH is associated with high mortality $(12.2 \%)$ and morbidity where majority of risk factors found in this study are preventable. 


\section{INTRODUCTION}

Postpartum hemorrhage (PPH) is defined as the blood loss of more than $500 \mathrm{mls}$ following vaginal delivery or more than $1000 \mathrm{mls}$ following caesarian section. It is primary PPH if is within 24 hours and secondary if is after 24 hours after delivery, however several factors such as initial haemoglobin level (HB) and infections should be considered. For instance secondary $\mathrm{PPH}$ most likely is associated with infections and may initially present with foul smelling vaginal discharge, subinvolution of uterus, pyrexia and tachycardia. [1, 2]

$\mathrm{PPH}$ is the leading cause of maternal mortality and morbidity world wide and it accounts for more than a quarter of all maternal mortality where as in parts of sub-Saharan Africa the proportion is even higher (Burkina Faso, 59\%; Ivory Coast, 37\%; Guinea, 43\%). [2]

Generally PPH is caused by atonic uterus, tissue retention, trauma and coagulopathy.

Uterine atony is the failure of the myometrium to contract and retract so that compresses torn blood vessels and control blood loss thus preventing dangerous hemorrhage which would otherwise ensue with horrifying speed.

It is caused by incomplete placental separation, retained placenta or membranes, precipitated labour, prolonged labor, polyhydramnious, multiple pregnancy, placenta preavia and abruption, general anaesthesia, mismanagement of the third stage of labor and a full bladder. Interfere with uterine contraction especially on completion of the second stage of labor is an important pathology in PPH. [3]

If bleeding occurs in spite the well contracted uterus and lack of retained tissues, then trauma of the uterus, vagina, cervix, perineum or labia may be suspected. External trauma can easily be suspected and repaired unlike internal trauma which is a bit difficult, but if bleeding remains uncontrolled surgery (hysterectomy) is inevitable. [3]

PPH can also be as a result of coagulation failure following severe pre-ecclampsia, antepartum hemorrhage, amniotic fluid embolus, intrauterine fetal death or sepsis. [4]

The usual presentation of PPH is one of the heavy vaginal bleeding that can quickly lead to signs and symptoms of hypovolaemic shock. Blood loss is usually visible at the introitus and this is especially true if the placenta has delivered. If the placenta remains in situ, then the significant amount of blood can be retained in the uterus behind the partially separated placental membranes.

PPH in the previous pregnancy is a major risk factor and every effort should be made to determine its severity and cause. Other predisposing factors are previous history of retained placenta, high parity resulting in uterine scar tissue, presence of fibroids, maternal anemia and ketoacidosis. [4, 5, 6]

PPH is unpredictable in up to $90 \%$ of cases and no identifiable risk factors are found nevertheless it has many potential causes as described above.

Furthermore, caesarian section delivery results in twice the average blood loss found in vaginal delivery as it is conducted in a poorly contractile lower segment though heal well. [7]

Clinicians should identify the risk factors before and during labor so that care may be optimized for high risk women. However significant life threatening bleeding can occur in absence of any risk factor and without any warning. All caregivers and facilities involved in maternal care must have a clear plan for prevention and management of PPH. This includes resuscitation skills and familiarity with all medical and surgical therapies available. 
WHO recommends the active management of the third stage of labour (AMTSL) with the uterotonics preferably oxytocin [2]. AMTSL can prevent about $60 \%$ of uterine atony and is evidencebasedfeasibleand low costintervention. However the use of oxytocin is limited due to the instability of it in tropical climates $[8,9,10]$ and lack of health professionals to administer injectables.

More importantly the greatest risk of maternal death is among women who deliver at home either alone or with tradition birth attendant (TBA) [9]. To date, effort to reduce maternal mortality through training TBAs have been disappointing [2].

\section{Study population and Methods:}

This study was a hospital based descriptive retrospective hospital based cross-sectional study among pregnant women from the day of admission, during delivery to after delivery. It was conducted in the labor ward of Mbeya referral hospital (MRH) in Mbeya region, southern Tanzania involving 344 pregnant women approaching delivery from July to midSeptember 2008.

The permission to conduct the study was sought from Muhimbili University of Health and Allied Science (MUHAS) and MRH Head of Department Obstetrics and Gynaecology through the Director of MRH. Participants were also informed and requested to participate either during or after admission but before the onset of active labour and those already in active labour were excluded to minimize errors. Objectives of the study were explained to participants and only those willing were included. A close ended Swahili checklist was used to get most of the information while for those already admitted some information obtained from register book. All information from participants was kept with confidentiality.

The data was analyzed and processed by using SPSS computer program version 10.0. Frequency distribution and cross-tabulation were done where chi-square was used to test statistical significance with p-value set at 0.05.

\section{RESULTS}

The prevalence of PPH among women delivered at MRH from July to September 2008 was $11.9 \%$ with $41.5 \%$ of women developed PPH end up into re-admission and $12.2 \%$ dead.

\section{Pie chat showing the outcome of women who developed PPH}
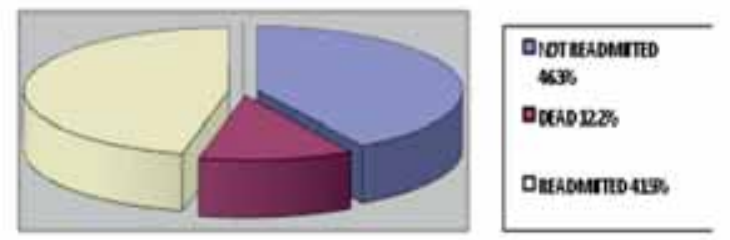

Majority (35.2\%) of women who developed PPH were transfused as an immediate measure. $43.9 \%$ of women had severe blood loss which was associated with the type of delivery LSCS being the leading cause of severe blood loss.

2. Bar chat showing association between the amount of blood loss and the type of delivery

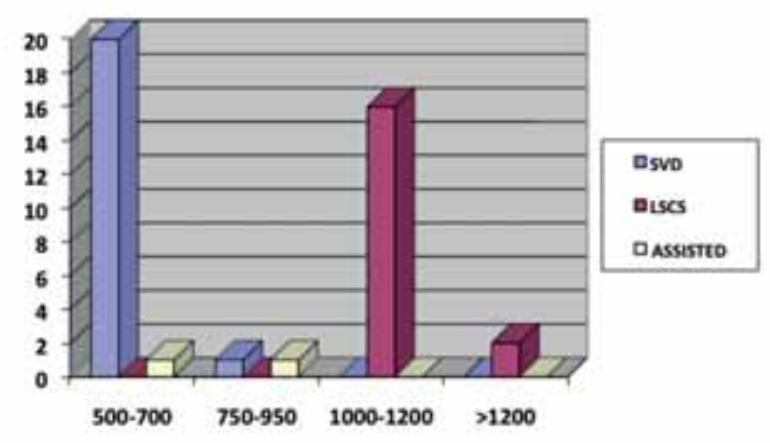

Key;SVD=spontaneous vaginal delivery

LSCS=lower segment caesarian section

Majority (56.2\%) of women with PPH presented with low $\mathrm{HB}$ and $\mathrm{PIH}$ prior to delivery, also $61 \%$ of women had trauma after delivery and BBA. 
3. Pie chat showing the complications found to the women developed PPH

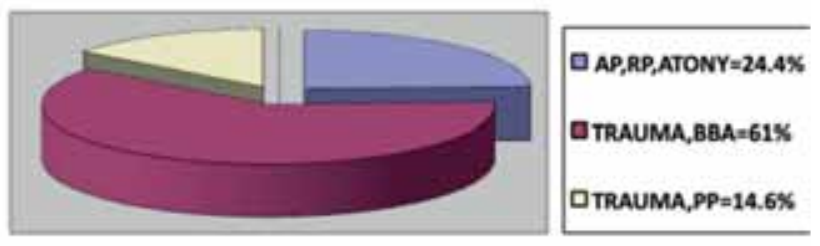

Key; $\mathrm{AP}=$ abruptio placenta

$\mathrm{PP}=$ placenta preavia

$\mathrm{BBA}=$ birth before arrival

A significant number of women (43.9\%) had not received any oxytocics after delivery and $\mathrm{PPH}$ seem to be associated with both parity (>para3) and advanced ( $>32$ years) maternal age.

\section{Bar chat showing age distribution among women who developed PPH}

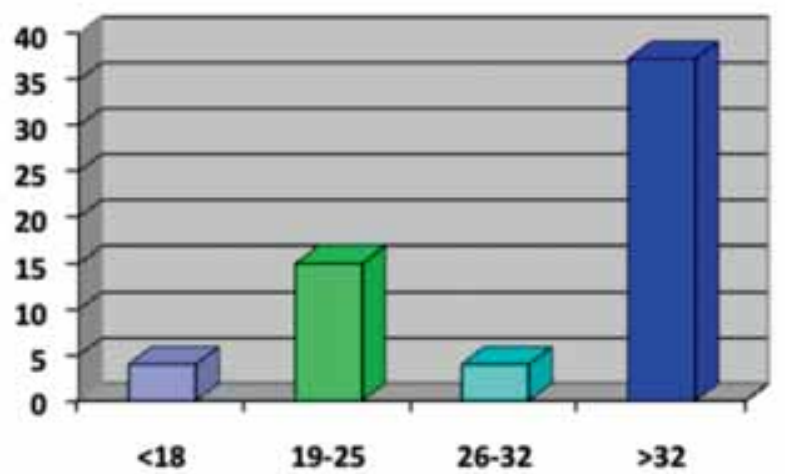

\section{Pie chat showing the parity among women developed PPH.}

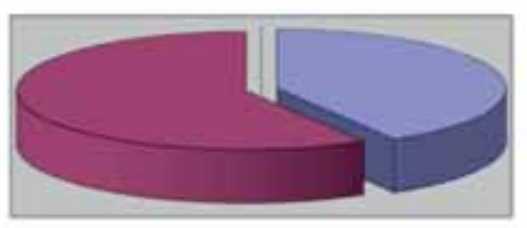

DPARA $1+241.5 \%$

DPARA $358.5 \%$

\section{DISCUSSION}

Tanzania health system puts pregnant women in special priority group for receiving essential health care especially maternal health care. Health education is given to majority of the women attending antenatal clinics with a special emphasis on safe way of delivery and risks identification before delivery. Early risks identification through antenatal clinic will reduce to some extent the prevalence of $\mathrm{PPH}$ in Tanzania. This study could not represent the actual problem throughout the country because data obtained from hospital are limited as are gathered to only those happen to come to hospital, however may provide valuable information.

From this research the prevalence of $\mathrm{PPH}$ in women delivered at Mbeya Referral hospital as well as re-admission rate seem to be low as compared to the general African prevalence and contrary to other studies done in Leicester UK, South Wales Australia and the University of Ilolin teaching hospital in Nigeria. [6,9] Many factors can account for this difference one being the level of economic status in these areas where the studies were conducted. (Developed world against developing world). Another difference can be seen in the methodology of the studies, either community based unlike the hospital based.

Also this research revealed high surgical and transfusion rate among women who developed PPH contrary to studies done in South Wales Australia, Department of Obstetrics and Gynaecology at Aga Khan hospital in Karachi Pakistan and Ilolin teaching Hospital in Nigeria. Severity of the problem gives different ways of management and both economic status and methodology used could also explain the difference seen between these findings. [8,9]

Further more, LSCS seems to be associated with high blood loss ( $>1000 \mathrm{mls})$ as compared with other types of delivery $(\mathrm{p}=0.02)$ and the common risks of developing PPH were found to be low $\mathrm{HB}$ and PIH in majority of the cases where others are trauma, BBA, advanced age (>32 years) and high parity (>para 3 ). These findings are the same to the study done by Ijaye in Nigeria but contrary to other study done in Leicester UK where majority were monoparous. $[6,9]$ 
A reasonable number of women who developed PPH (43.9\%) found not used any oxytocic during and after delivery explained by a number of women who had BBA. Oxytocin was the major type of oxytocic used in MRH which may be due its availability and few side effects as compared to other oxytocics. Furthermore, $\mathrm{MRH}$ is an expert institution so we expect better services to patients. The use of oxytocin as the major oxytocic is different to the study done by WHO in Kigoma involving trained traditional birth attendants where misoprostol was the oxytocic of choice. [11]

PPH seem to be associated with high proportional of maternal mortality (12.2\%) which is also documented in other previous studies already done in Africa and developed world.

\section{CONCLUSSION}

The prevalence of PPH was found to be $11.9 \%$ with reasonably high mortality, however seems to be lower as compared to overall for Africa and a reasonable number of women were re-admitted where surgery and blood transfusion were taken as an immediate mode of management.

LSCS was found to be associated with large amount of blood loss $(\mathrm{p}=0.02)$ where there is still large number of women delivered before arrival to health facilities, the reason accounting for high prevalence value PPH in developing countries.

Risk factors for developing PPH found in this study are all preventable only if more effort is directed to this problem.

\section{ACKNOWLEDGEMENT}

School of public health and social science at MUHAS, Government of the Republic of Tanzania through the Ministry of education and Higher learning, Dr P Wangwe (Department of Obstetrics and Gynaecology,MNH) and the administration of MRH and staffs for their support.

\section{REFERENCE}

1. V.Ruth Bennett (ed), Myles text book of Obstetrics, 12th edition; 1993 pg 462470.

2. WHO. Mother-Baby package: implementing safe motherhood in countries. Geneva 7 World Health Organization; 1994.

3. AbouZahr C. Antepartum and postpartum hemorrhage. In: Murray CaAL editor. Health dimensions of sex and reproduction. Boston 7 Harvard School of Public Health on behalf of the World Bank and the World Health organization.

4. Fortney JA, Susanti I, Gadalla S, Saleh S, Rogers SM, potts M. reproductive mortality in two developing countries. Am J Public Health 1986; 76(2):134-8

5. Maine D, Rosefield A. the safe motherhood initiative: why has it stalled? Am J Public Health 1999; 89(4):480-2

6. Hazra, Chilaka VN, Rajendran S, Konje JC. Massive postpartum hemorrhage as a cause of maternal morbidity in a large tertially hospital. J Obstetrica and Gynaecology.2004 Aug; 24(5):519-20

7. Cameron CA, Roberts CL, Olive EC, Ford $\mathrm{JB}$, fischer WE. Trends in postpartum hemorrhage. Aust NZJ Public Health.2006 Apr; 30(2):151-6.

8. Sheikh L, Zuberi NF, Riaz R, Rizvi JH. Massive primary post partum hemorrhage: setting up standards of care. J Pak Med Assic.2006 Jan; 56(1):26-31.

9. Ijaiya MA, Aboyeji AP, Abubakar D. massive postpartum hemorrhage as a cause of maternal morbidity in a large tertially Hospital. J Obstet Gynaecol. 2003 Jul; 23(4):374-7

10. Prata N, Mbaruku G, Campbell M, Potts M, Vahidnia F. controlling postpartum hemorrhage after home births in Tanzania. Int J Gynaecol Obstet.2005 Jul; 90(1):51-5. 